1999-01-01

\title{
A cloud-removal algorithm for SSM/I data
}

David G. Long

david_long@byu.edu

Douglas L. Daum

Quinn P. Remund

Follow this and additional works at: https://scholarsarchive.byu.edu/facpub

Part of the Electrical and Computer Engineering Commons

\section{Original Publication Citation}

Long, D. G., Q. P. Remund, and D. L. Daum. "A Cloud-Removal Algorithm for SSM/I Data."

Geoscience and Remote Sensing, IEEE Transactions on 37.1 illustrated through simulation and real data distribution analysis. The results show that the second-highest value algorithm is biased high. MMA provides a more accurate brightness temperature estimate in areas of atmospheric distortion, whil(TRUNCATED) (1999): 54-62

\section{BYU ScholarsArchive Citation}

Long, David G.; Daum, Douglas L.; and Remund, Quinn P., "A cloud-removal algorithm for SSM/I data" (1999). Faculty Publications. 631.

https://scholarsarchive.byu.edu/facpub/631

This Peer-Reviewed Article is brought to you for free and open access by BYU ScholarsArchive. It has been accepted for inclusion in Faculty Publications by an authorized administrator of BYU ScholarsArchive. For more information, please contact ellen_amatangelo@byu.edu. 


\title{
A Cloud-Removal Algorithm for SSM/I Data
}

\author{
David G. Long, Senior Member, IEEE, Quinn P. Remund, and Douglas L. Daum
}

\begin{abstract}
Microwave radiometers, while traditionally utilized in atmospheric and oceanic studies, can also be used in land surface applications. However, the problem of undesirable atmospheric effects caused by clouds and precipitation must be addressed. In this paper, temporal composite surface brightness images are generated from special sensor microwave/imager (SSM/I) data with the aid of new algorithms to eliminate smallscale distortion caused by clouds or precipitation. Mean, secondhighest value, modified maximum average (MMA), and hybrid compositing algorithms are compared. The effectiveness of each algorithm is illustrated through simulation and real data distribution analysis. The results show that the second-highest value algorithm is biased high. MMA provides a more accurate brightness temperature estimate in areas of atmospheric distortion, while the mean is superior in regions with little or no distortion. A hybrid algorithm is developed that is a combination of MMA and mean. It utilizes the strengths of both to create a superior algorithm for regions with varying levels of distortion. Uses of composite images produced by these algorithms include studies of vegetation change, land cover classification, and surface parameter extraction.
\end{abstract}

Index Terms - Cloud removal, compositing, electromagnetic atmospheric interference, microwave radiometry.

\section{INTRODUCTION}

$\mathbf{M}$ ICROWAVE radiometers, such as the special sensor microwave/imager (SSM/I) [5], [6] have wide application in atmospheric remote sensing over the ocean and provide essential inputs to numerical weather prediction models. SSM/I data have also been used for land and ice studies, including measurements of snow cover classification [4], soil and plant moisture content [8], [15], atmospheric moisture over land [10], land surface temperature [12], and mapping polar ice [18].

Because the surface brightness observed by the SSM/I may be adversely affected by spatial variations in the atmospheric profile over the surface, algorithms for cloud removal have been developed [1], [10]. In this paper, we compare several new algorithms that generate cloud-free composite images from multiple passes of the study region. Simulations to determine the effectiveness of these algorithms are performed. Actual SSM/I data are analyzed by exploring the effects of compositing algorithms on the pixel surface brightness temperature distributions. This paper is organized as follows. After a brief background discussion in Section II, Section III discusses the production of no-cloud composite images. Section IV introduces the modified maximum average (MMA) and hybrid

Manuscript received November 18, 1996; revised October 27, 1997.

The authors are with Brigham Young University, Provo, UT 84602-4099

USA (e-mail: long@ee.byu.edu).

Publisher Item Identifier S 0196-2892(99)00024-8.
TABLE I

SSM/I CHANNELS

\begin{tabular}{c|c|c|c}
\hline \multicolumn{2}{c|}{ SSM/I Channel } & \multicolumn{2}{c}{3 dB Footprint (km) } \\
Frequency (GHz) & Pol. & Along-track & Cross-track \\
\hline 19.35 & $\mathrm{~V}$ & 69 & 43 \\
\hline 19.35 & $\mathrm{H}$ & 69 & 43 \\
\hline 22.235 & $\mathrm{~V}$ & 50 & 40 \\
\hline 37.0 & $\mathrm{~V}$ & 37 & 28 \\
\hline 37.0 & $\mathrm{H}$ & 37 & 29 \\
\hline 85.5 & $\mathrm{~V}$ & 15 & 13 \\
\hline 85.5 & $\mathrm{H}$ & 15 & 13 \\
\hline
\end{tabular}

algorithms. A simulation experiment to compare the cloudremoval algorithms is presented in Section V. Section VI discusses the analysis of actual SSM/I data. Finally, the conclusions are given.

\section{BACKGROUND}

The SSM/I is a total-power, seven-channel, four-frequency radiometer [5]. The channels are horizontal and vertical polarizations at $19.35,37.0$, and $85.5 \mathrm{GHz}$ and vertical polarization at $22.235 \mathrm{GHz}$. It utilizes an integrate-and-dump filter as the antenna scans the ground track [7]. The 3-dB antenna footprints range from about 15 to $70 \mathrm{~km}$ in the along-track direction and 13 to $43 \mathrm{~km}$ in the cross-track direction (see Table I). The 3-dB antenna footprints, which are different for each frequency, generally have an elliptical shape on the surface of the earth due to the elevation angle of the radiometer [6].

The brightness temperatures observed by the SSM/I are a function of the effective brightness temperature of the earth's surface and the emission, scattering, and attenuation of the atmosphere. Because of the spatial and temporal variability of the surface brightness, which is a function of the properties of the soil and overlaying vegetation and their physical temperatures, it is difficult to decompose the observed brightness into its individual components. The most crucial factors affecting a radiometric measurement, however, are the surface emissivity and temperature, the vegetation canopy, and the atmospheric conditions [19].

\section{Generation of Cloud-Free Images}

One of the challenges in mapping the surface brightness from spaceborne radiometer data is atmospheric distortion. Cloud cover and precipitation are two primary sources of this distortion. Although cloud and rain cause little microwave attenuation for frequencies less than $10 \mathrm{GHz}$, the higher microwave frequencies of the SSM/I $(19.35,22.235,37.0$, and $85.0 \mathrm{GHz}$ ) show substantial atmospheric loss due to scattering 


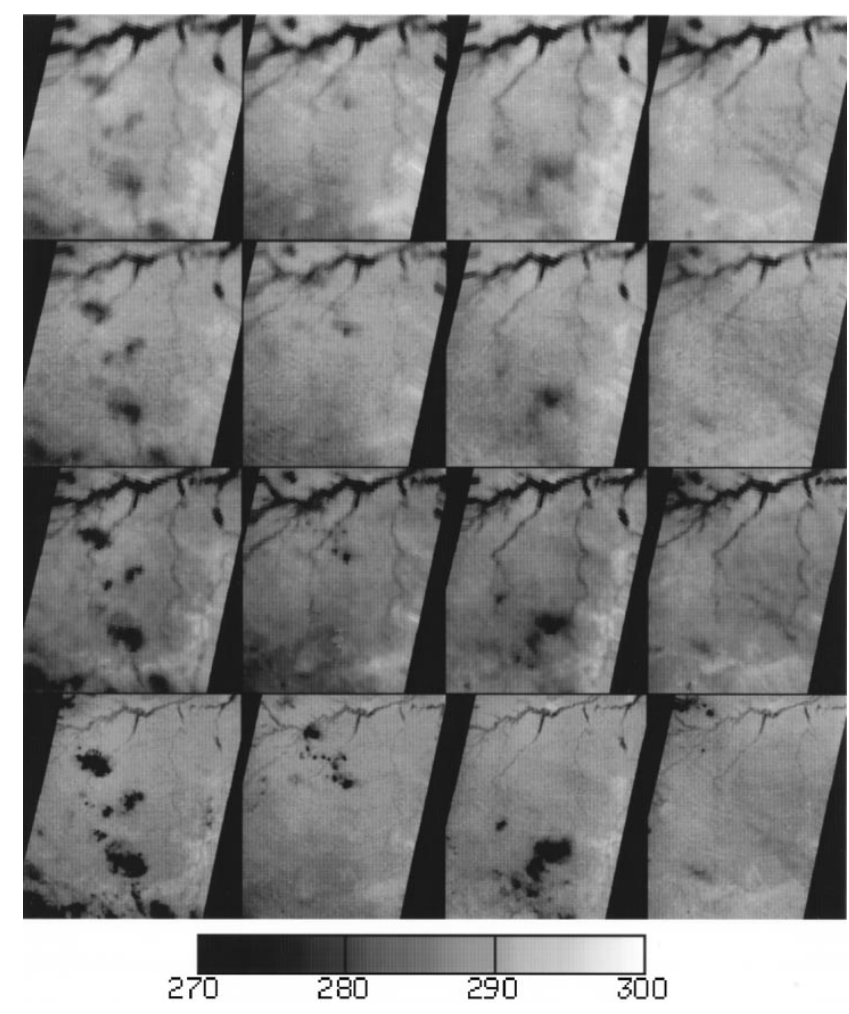

Fig. 1. Individual SSM/I swath examples of temporal atmospheric distortions. These images were created by assigning the closest measurement value to each pixel. Columns (from left to right) correspond to 1992 Julian Days 245, 248, 261, and 264 of the passes. Rows (from top to bottom) indicate SSM/I channels $19 \mathrm{~V}, 22 \mathrm{~V}, 27 \mathrm{~V}$, and $85 \mathrm{~V}$.

from hydrometeors and water vapor. Over the ocean, the atmospheric signal is used to deduce cloud water content from the change in brightness. For studies of the land surface, however, these atmospheric effects may be unwanted [17].

Clouds and precipitation affect surface brightness measurements in two ways. First, the cloud scattering nonuniformly lowers the measured brightness temperature for all frequencies of the SSM/I with higher frequencies progressively more sensitive. The reduction in brightness temperature can be confused with surface features. Second, the clouds attenuate the polarization differences caused by the geometric or chemical composition of different surface types. This prevents the surface polarization difference from being used to discriminate between vegetation types and/or standing water.

Fig. 1 illustrates examples of atmospherically distorted brightness temperature images in a region of the Amazon Basin for all vertical polarization SSM/I channels. These images, like those in this paper, were generated by assigning to each pixel covered by the swath the value of the nearest measurement. Other single-pass imaging techniques can also be used, e.g., [2] and [11]. The distortions are evident in the temporal variation of surface brightness temperature in different areas. Note that, as expected, the distortions are more pronounced in the higher frequency channels. This follows the trend of increased atmospheric scattering due to clouds and precipitation with increasing frequency. The distortions of pixel values can be as high as $60 \mathrm{~K}$ for the higher frequency channels. These distortions can greatly hinder the application of SSM/I data to land studies.

While multichannel- and/or multisensor-based algorithms for cloud removal have been previously used (e.g., [4], [14], [16], and [17]), we use a single-channel algorithm similar to [1]. By using only single-frequency information to generate a "cloud-free" image of the surface, we avoid introducing spurious correlation between the channels. For example, since each frequency has a different footprint size, using lower frequency data to remove atmospheric distortion effects in higher frequency channels may unnecessarily exclude undistorted values in the higher frequency channels [1].

Our algorithm is based on the assumption that temporal surface brightness variations over an area are caused by smallscale atmospheric effects rather than temporal changes in the surface brightness. Using multiple passes over the surface, we generate a composite image that represents the effective surface brightness temperature over a multiday period. The composite image is generated from images created from each descending pass, though ascending passes can also be used.

In the example data that follow, 20 days of descending pass SSM/I data (September 1992) over South America are used. During this period, each pixel is observed from five to ten times. The value of the composite pixel is computed from this ensemble. The study region is considered a worst-case example, with frequent rain and distortion events occurring up to several times during the compositing interval. For this region, 20 days offers a good balance between the number of undistorted measurements in the ensemble and temporal variations due to seasonal radiometric surface response variations. This is some what less than the 30 days used by previous investigators [1], but it provides adequate results. Areas with less-frequent distortion events may be able to use shorter periods.

Choudhury and Tucker [1] removed temporal atmospheric distortion by using the second-highest pixel value from the ensemble as the composite pixel value. Since the atmospheric distortion generally lowers the brightness temperature measurements over land, high pixel values have the least atmospheric influence. They reason that, since the highest value is often strongly influenced by noise or processing artifacts, they used the second-highest pixel value.

Choosing the second-highest value is an example of a rank order statistic [3]. Another rank order technique is the median filter [9]. As an estimator, a rank order statistic is noisereducing, but it is sensitive to the underlying distribution of the samples [13]. Thus, the second-highest value technique's ability to reduce noise is strongly influenced by the measurement distribution.

Unfortunately, the distribution function for the SSM/I data is not precisely known and it is not possible to analytically determine the estimator variance. However, it is known that in the presence of atmospheric distortion over land, the distribution is skewed low, while the desired estimation parameter is the mode on the high end of the distribution [14]. This strongly suggests that the rank order statistic needed for this application is a value closer to the highest value than to the median value. Given this insight, the second-highest value method is a reasonable approach. 


\section{MMA ALgORIthM}

In an effort to improve the performance of a cloud-removal algorithm, an MMA technique was developed. This algorithm attempts to estimate the cloud-free surface brightness of a pixel by choosing a subset of pixel values from the ensemble of measurements of that pixel and then averaging the selected values together. By properly selecting the subset from the ensemble, the cloud distortion is eliminated. Averaging of the subset reduces the noise and attenuates any residual bias.

To select pixel values from the ensemble in the MMA technique, the sample mean of the entire pixel ensemble is first computed. Measurements greater than the sample mean yield a subset of the complete ensemble corresponding to its highest values. The highest value of this subset is then eliminated. The remaining values consist of those values that are above the ensemble mean but less than the maximum value of the ensemble. This is the MMA subset. The estimated pixel value is then determined as the mean of this subset.

Analyzing this technique statistically is challenging for two reasons: 1) the distribution of pixel values when distortions are included is not clearly known and 2) the algorithm combines both box averaging statistics and order statistics. To qualitatively justify this approach, consider a simple model for the pixel measurements. In this model, the measurement is the sum of a Gaussian-distributed surface brightness temperature and a weighted binary random variable

$$
z=\eta(\mu, \sigma)-p_{d} \Delta
$$

where $z$ is the measured brightness temperature, $\eta$ is the Gaussian distribution with mean $\mu$ and standard deviation $\sigma$, $p_{d}$ is a binary-valued random variable of the probability that a measurement contains cloud distortion (less than $30 \%$ based on a simple examination of SSM/I in the study region described later), and $\Delta$ is a positive random variable representing the drop in brightness temperature due to a cloud ( $\Delta$ depends on the cloud thickness, water content, etc., the statistics of which are unknown). A schematic example of the distribution of $z$ is given in Fig. 2(a), where $\Delta=10 \mathrm{~K}$ and $p_{d}=30 \%$. The right mode corresponds to the distribution of surface brightness, while the lower mode represents the distribution of cloudy pixels. The " $\mathrm{X}$ " marks below the temperature axis illustrate an ensemble of seven random measurements for a given pixel. Also illustrated are the results from applying the MMA and second-highest value techniques.

Good metrics for comparing estimation algorithms include the mean estimate error (bias) and the estimate variance. Ideally, the estimate should have no bias and minimum variance. To compare the variances of the MMA algorithm and the second-highest value technique, consider Fig. 2(b). As in Fig. 2(a), the X's represent an ensemble of seven samples taken from the distribution. The variance of the second-highest value technique is governed by the average temperature difference between the highest and third highest value of the ensemble. The variance of the MMA algorithm depends on the variances of the second, third, and fourth measurements. Graphically, we may see that the averaging of these values lowers the estimate variance more than just using the secondhighest value.

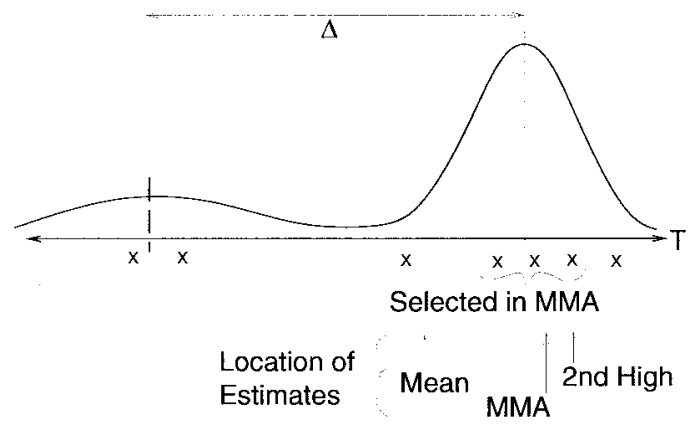

(a)

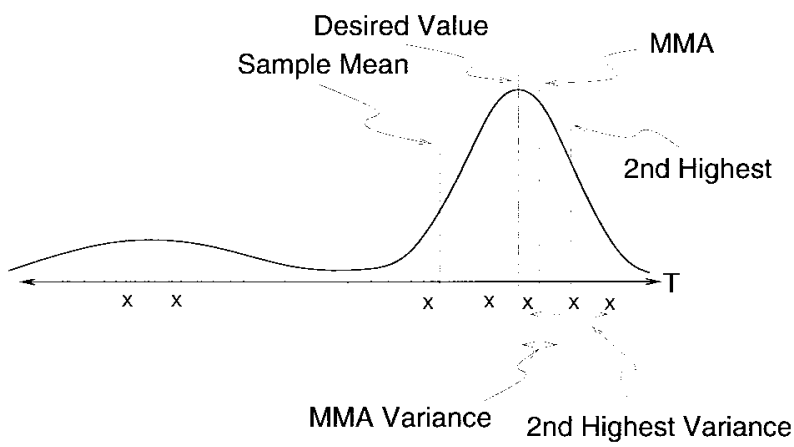

(b)

Fig. 2. Illustration of hypothetical radiometric measurement distribution for a cloudy region. (a) Sample discrete ensemble. (b) Variance of the modified maximum and second-highest value.

Like the second-highest value estimate, the MMA estimate in this example is biased high, and it is high whenever the ensemble includes more than one sample from the lower mode of the mixture distribution. However, it is clear that the MMA bias is less than the second-highest value estimate. Further, the estimator variance is smaller for MMA.

While MMA produces a less-biased estimate for pixels with high cloud contamination than the second-highest value, it is still biased high for pixels with little or no contamination. Fig. 3 depicts a hypothetical distribution of brightness temperatures for a noncloud-affected pixel. In this case, the second-highest value and MMA estimates are biased high. The desired value is the mean of the overall distribution in the absence of clouds or precipitation. As previously noted, a simple examination of SSM/I data reveals a probability of less than $30 \%$ that a measurement is distorted by clouds or rain. Thus, MMA may be unnecessarily biased somewhat high for many of the measurements.

In an effort to ameliorate this problem, a hybrid of the mean and MMA methods has been developed. Ideally, this hybrid implements MMA in the presence of clouds and takes the mean in their absence. This reduces the overshoot of MMA for low atmospheric distortions and provides a better estimate of the actual surface brightness temperature.

To implement the hybrid algorithm, a metric is required for the decision making process. The chosen metric is the temporal standard deviation of the values for a particular pixel. The presence of clouds skews the brightness temperature distribution low for affected passes, thus increasing the standard deviation. Fig. 4 shows a mean SSM/I composite image along with 

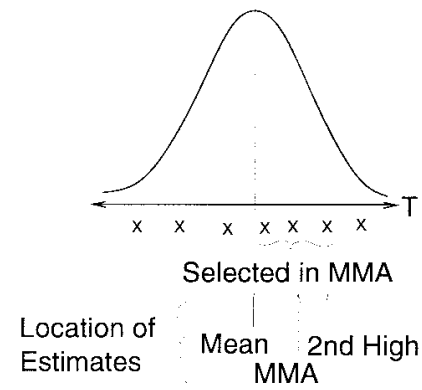

(a)

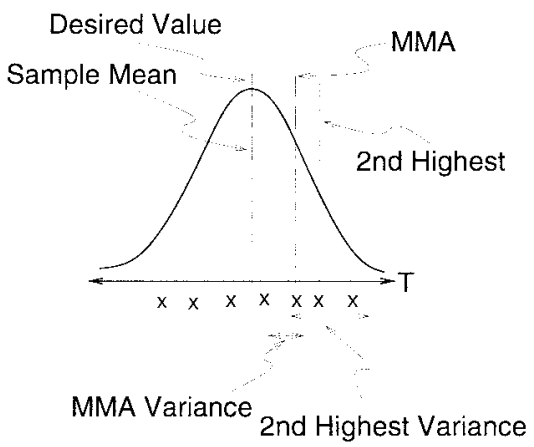

(b)

Fig. 3. Illustration of hypothetical radiometric measurement distribution for a clear region. (a) Sample discrete ensemble. (b) Variance of the modified maximum and second-highest value.

its corresponding temporal standard deviation image for the $85-\mathrm{GHz}$ vertically polarized channel. This visually illustrates that the standard deviation highly correlates with atmospheric distortions. Areas that appear darkened in the mean composite image exhibit relatively high values in the standard deviation image.

In the standard deviation image of Fig. 4, the areas with low values correspond to regions with little or no atmospheric distortion. A small $2 \times 2^{\circ}$ spatially homogeneous subregion, which will be more explicitly defined in a later section, is chosen as an example of an area with low temporal variation and thus low atmospheric distortion. The temporal mean and standard deviation of all swath pixel values are calculated for each vertically polarized SSM/I channel in this subregion and shown in Table II. The standard deviations represent the temporal variance of surface brightness temperature in the absence of atmospheric distortion. According to the previous discussion, any kind of temporal variation, such as atmospheric distortion, will cause the standard deviation to rise above these values. All channel standard deviation values are similar with the $19-\mathrm{V}$ channel exhibiting the highest and the $37-\mathrm{V}$ channel the lowest. Ideally, optimum values should be used for each channel in implementing the hybrid algorithm. However, since the temporal standard deviations are similar, and for the sake of simplicity, we chose to use the highest of these values $1.25 \mathrm{~K}$ as the hybrid threshold metric for the results presented in this paper. In the hybrid algorithm, the standard deviation is computed for each pixel ensemble of brightness temperatures. If it is above $1.25 \mathrm{~K}$, the MMA algorithm is used to produce the composite value for that particular pixel to select only nondistorted measurements. Otherwise, the mean is used. We note that this threshold has been chosen for use in the study region and should be tuned for other regions.

\section{SimUlation}

To further compare and contrast the mean, second-highest value, MMA, and hybrid algorithms, a simple Monte Carlo analysis for a single pixel is presented. This simulation assumes that the true pixel brightness for a geographical area is $280 \mathrm{~K}$. An ensemble of seven pixel values is then created by adding a Gaussian random variable of standard deviation $1 \mathrm{~K}$ to the "true" value. This represents the radiometric "noise" $\Delta T$ inherent to the radiometer measurements. Seven pixels simulate the average number of radiometric measurements in the 20-day study period. Two of the ensemble measurements then have simulated atmospheric distortion added. The first measurement is reduced by $T_{\mathrm{dis}}$ and the second measurement by $T_{\text {dis }} / 2$. This models a pixel that is contaminated by clouds at two different times with one cloud twice as distorting as the other. The seven-member ensemble is then processed by each algorithm, and the results are saved. The results of 1000 simulations are then analyzed to give the results in Fig. 5. For comparison, the ensemble mean is plotted along with the windowed mean. The windowed mean is the mean of values within one standard deviation of the ensemble mean.

For pixels with little or no atmospheric distortion, the mean or windowed average is closer to the 280 "true" value than MMA or the second-highest value. For ensembles that have greater $(>5 \mathrm{~K})$ atmospheric distortion, the second-highest value and MMA techniques are superior. The MMA technique has the smallest bias of the two. Since it also has the smallest variance, the MMA algorithm is considered superior to the second-highest value algorithm. The hybrid algorithm combines the strengths of the mean method for low distortion temperatures and MMA for high distortion temperatures. This is evident in Fig. 5 by the closer estimates to $280 \mathrm{~K}$ for small $T_{\text {dis. }}$. The simulation results indicate that MMA is superior in the presence of significant distortion and mean is best with little or no distortion present, while the hybrid algorithm combines the two in a manner that uses the appropriate algorithm for each pixel.

\section{SSM/I DATA ANALYSIS}

To validate the algorithms with actual data, a region of the Amazon Basin was chosen for SSM/I data analysis. The region lies primarily within Brazil and is bounded by the coordinates: $48-63^{\circ} \mathrm{W}$ longitude and $1-16^{\circ} \mathrm{S}$ latitude. Its characteristic high precipitation levels make it a good study region, representing a worst-case scenario with frequent rain and distortion events. The mean, second-highest value, MMA, and hybrid composite images of this region were created for all vertically polarized SSM/I channels. Examples are presented in Figs. 6 and 7. In the interest of space, only the 37- and $85-\mathrm{V}$ images are shown here. Due to smaller 3-dB antenna footprints, the higher frequency images exhibit better effective resolution. 

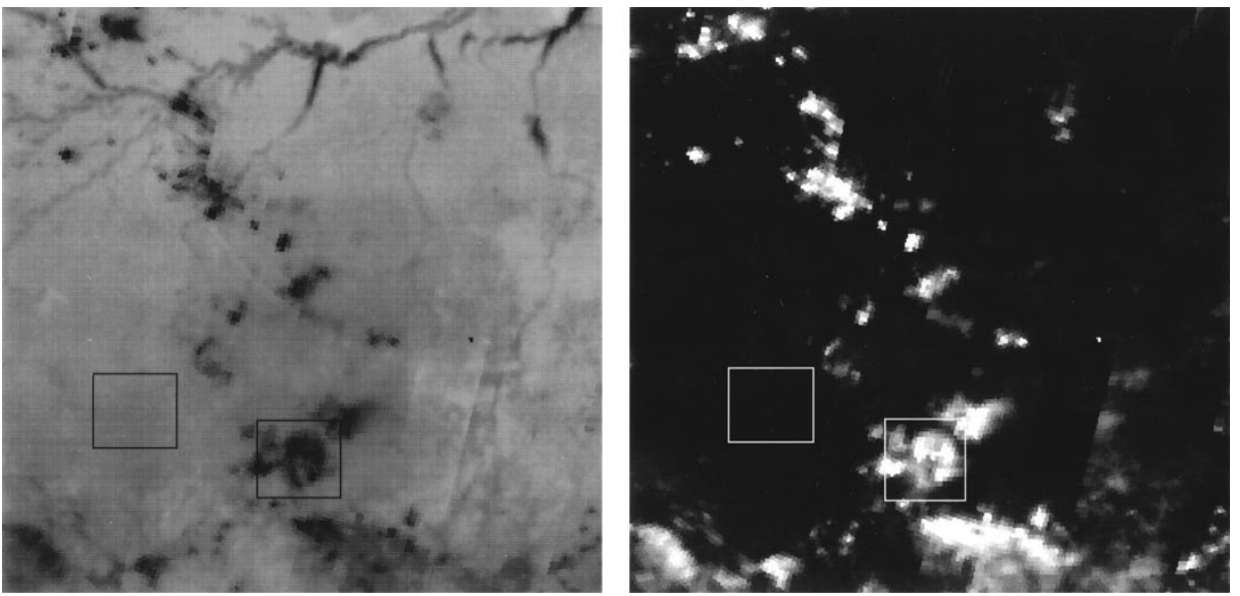

Fig. 4. Temporal mean and standard deviation images of the $85-\mathrm{GHz}$ vertically polarized SSM/I channel. These were generated using 44 descending single-pass images corresponding to days $245-264$ of 1992 . The left panel is the mean image, and the right panel is the standard deviation image. The boxes define two $2 \times 2^{\circ}$ subregions used later in the assessment of algorithm effectiveness. One is an example of a cloudy region, and the other is a region with little or no distortion. The band in the lower-right area of the standard deviation image results from atmospheric distortions affecting only some passes.

TABLE II

Temporal Means and Standard Deviations of Pixel Brightness Temperatures in a $2 \times 2^{\circ}$ Distortion-Free Subregion of the Amazon Basin

\begin{tabular}{c|c|c}
\hline Channel & Mean & Std. \\
\hline $19 \mathrm{~V}$ & 287.2 & $1.25 \mathrm{~K}$ \\
\hline $22 \mathrm{~V}$ & 284.9 & $1.23 \mathrm{~K}$ \\
\hline $37 \mathrm{~V}$ & 283.2 & $1.14 \mathrm{~K}$ \\
\hline $85 \mathrm{~V}$ & 284.6 & $1.20 \mathrm{~K}$ \\
\hline
\end{tabular}

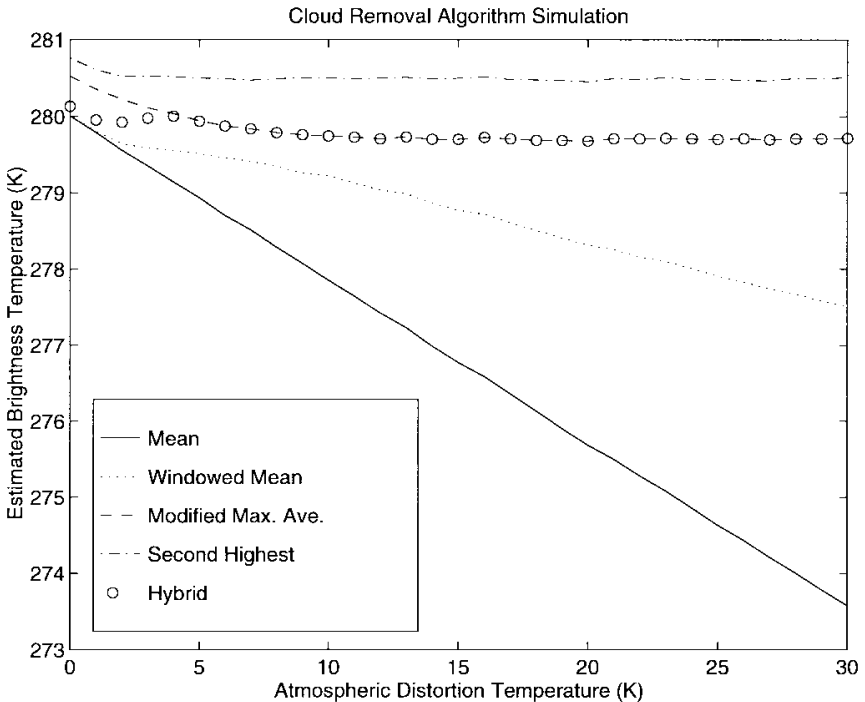

Fig. 5. Simulation results of atmospheric distortion removal (true value is $280 \mathrm{~K})$.

Two small nearly spatially-homogeneous regions were chosen from the larger study region for brightness temperature distribution analysis. Each is $2^{\circ}$ square in latitude and longitude. The first region, with bounding coordinates of 54.5-56.5 ${ }^{\circ} \mathrm{W}$ longitude and $11.5-13.5^{\circ} \mathrm{S}$ latitude, exhibits temporal variations consistent with atmospheric distortion. The second was chosen for its apparent absence of cloud or precipitation distortion with boundaries of $58.5-60.5^{\circ} \mathrm{W}$ longitude and $10.5-12.5^{\circ} \mathrm{S}$ latitude. Both subregions are shown in Fig. 4.

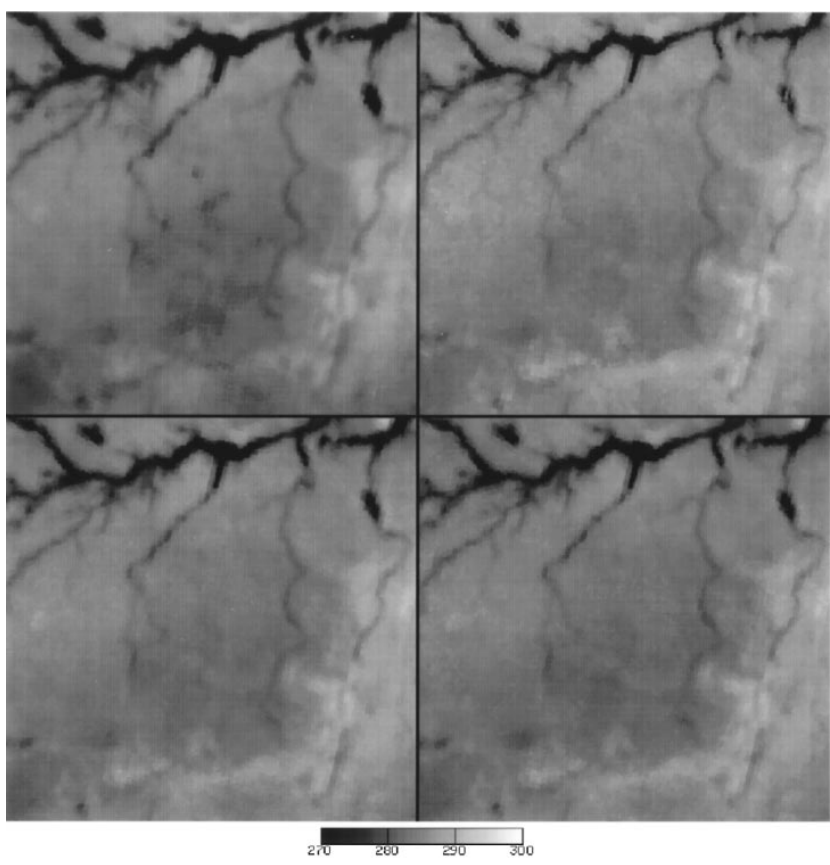

Fig. 6. SSM/I 37-V Brazil region composite images. From top left to bottom right: mean, second-highest value, MMA, and hybrid.

The brightness temperature distribution of all SSM/I swath measurement data over the small regions is presented in Figs. 8 and 9. These help validate the distortion model used previously. Fig. 8 depicts distributions for the cloudy region, and Fig. 9 shows the same for the clear region. Note that a log vertical scale has been used to emphasize the distribution tails. These distributions demonstrate the difference between cloudy and clear regions. The cloudy region distribution has a relatively wide peak at the high end with a long tail of lower temperatures. The peak corresponds to undistorted brightness temperatures, while the tail represents pixels that have experienced some level of atmospheric distortion. We consider only the $85-\mathrm{GHz}$ channel for the rest of the analysis for conciseness. 


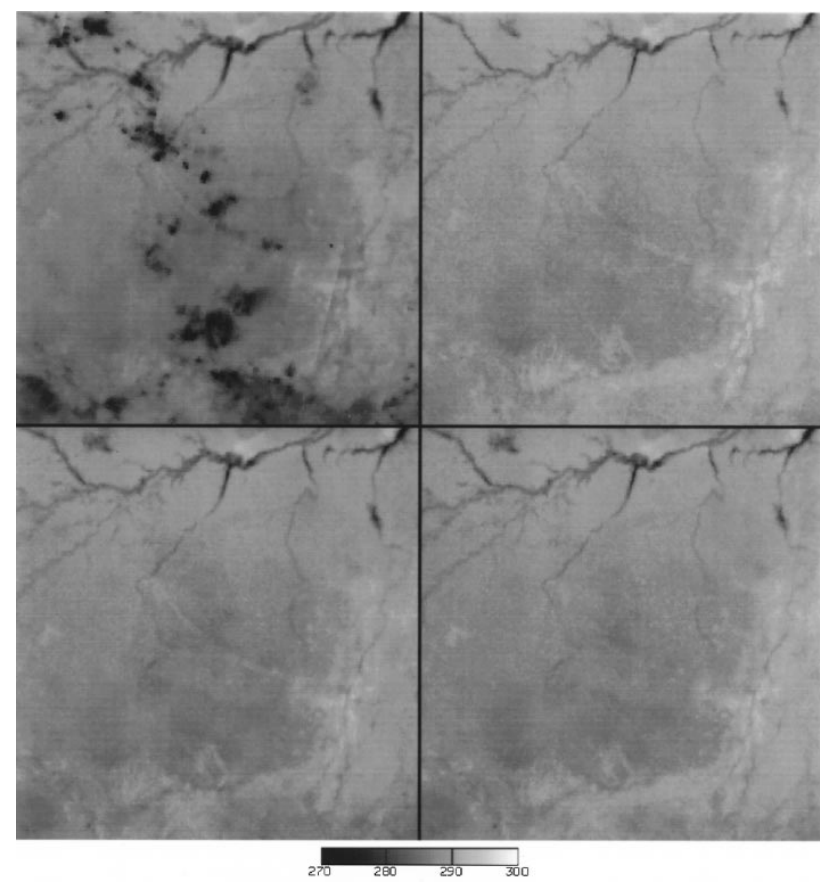

Fig. 7. SSM/I 85-V Brazil region composite images. From top left to bottom right: mean, second-highest value, MMA, and hybrid.
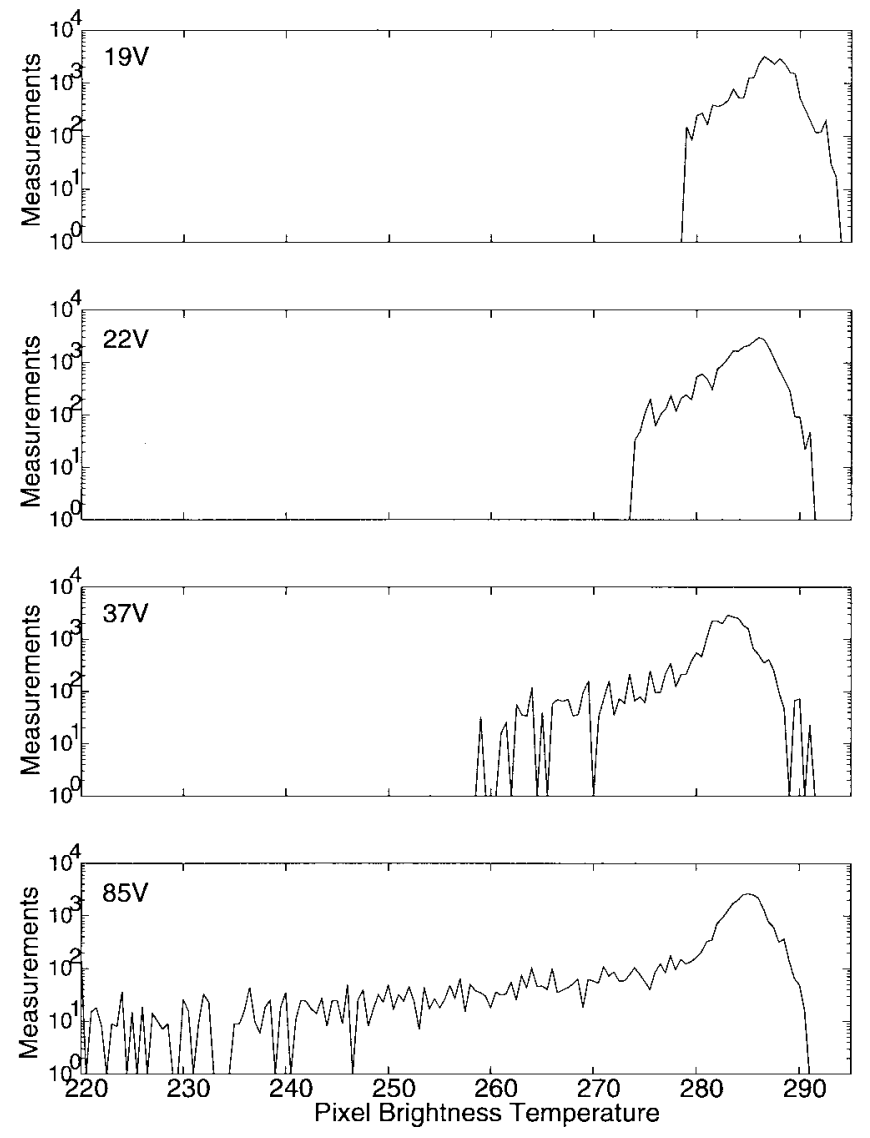

Fig. 8. Cloudy region temporal and spatial measurement histograms for all SSM/I vertically polarized channels. A log vertical scale has been used to emphasize the tails of the distributions. These tails correspond to atmospheric distortions that lower the overall brightness temperature.
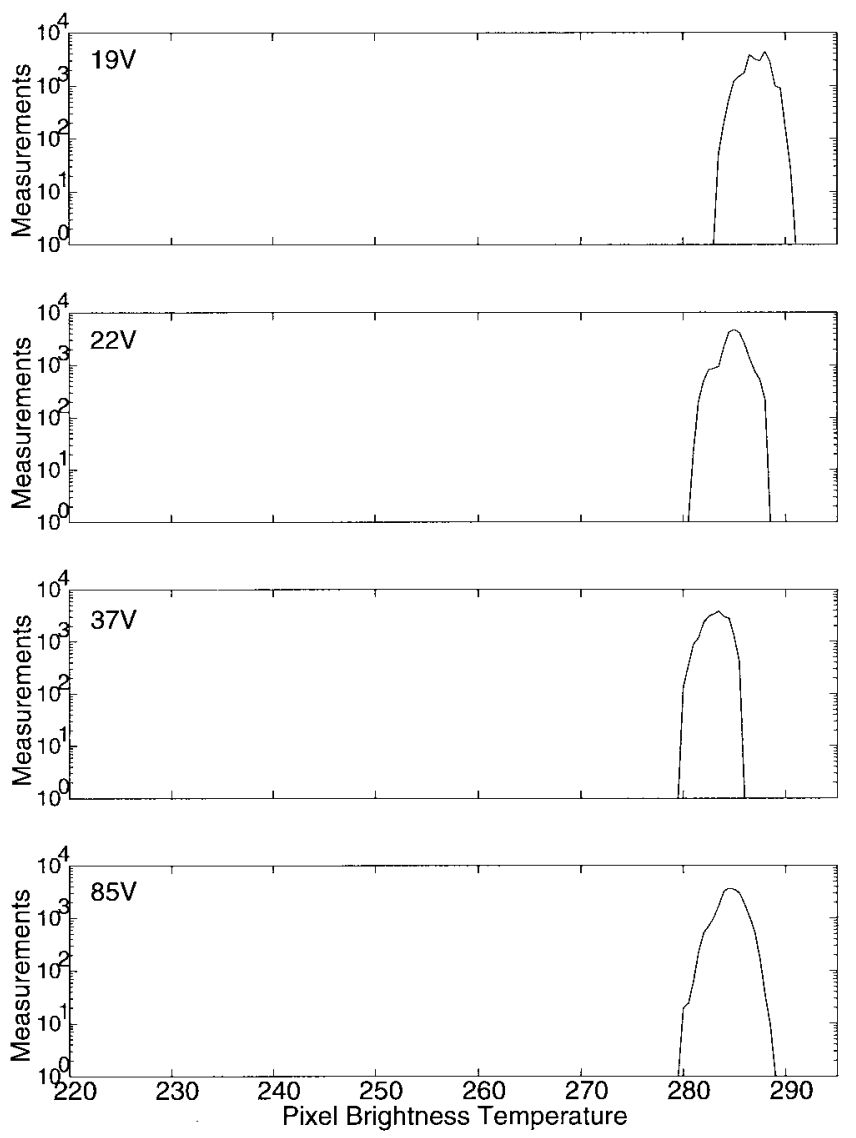

Fig. 9. Clear region temporal and spatial measurement histograms for all SSM/I vertically polarized channels. Compare these distributions with those in Fig. 10. The lack of tails on the distributions indicates the absence of significant atmospheric distortions.

The clear region has a clean single-modal distribution with a tight peak and no trailing tail, indicating the absence of any distortion. Hence, the effect of atmospheric distortion on the surface brightness distribution is to widen the upper modal peak and add a lower tail of brightness values. A good cloudremoval algorithm would select only values within the mode that represent the undistorted brightness temperatures.

Images for both subregions were created using the mean, second-highest value, MMA, and hybrid algorithms. These images are shown in Figs. 10 and 11. Since no surface brightness temperature ground truth is available for validation of the estimated surface brightness temperature, it is difficult to objectively assess the effectiveness of each algorithm. However, a visual interpretation of these images yields some important information.

Fig. 10 illustrates each composite image for the cloudy region at $85-\mathrm{GHz}$ vertical polarization. Here, the presence of clouds and precipitation is evident. Certainly, it can be seen that the second-highest value, MMA, and hybrid algorithms all perform superior to mean. The distortions are still evident in the mean composite image, while the others appear to remove the distortions to some degree. The average and standard deviation of pixel values were computed for each composite image. The results are given in Table III. Without knowledge of the true surface brightness temperature, it is not 


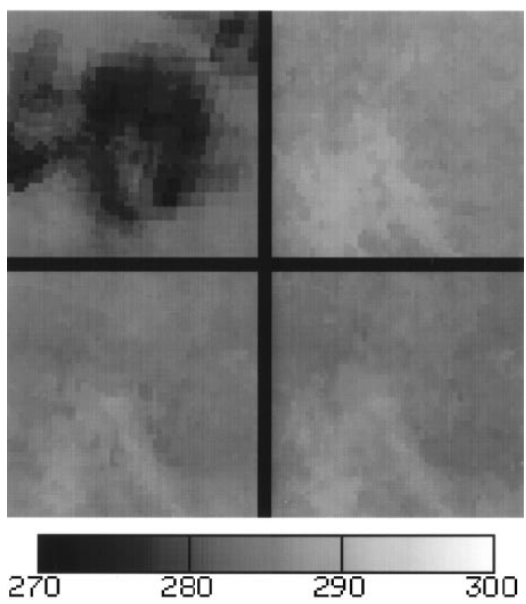

Fig. 10. SSM/I 85-V cloudy region cloud-removal composite images. From top left to bottom right: mean, second-highest value, MMA, and hybrid.

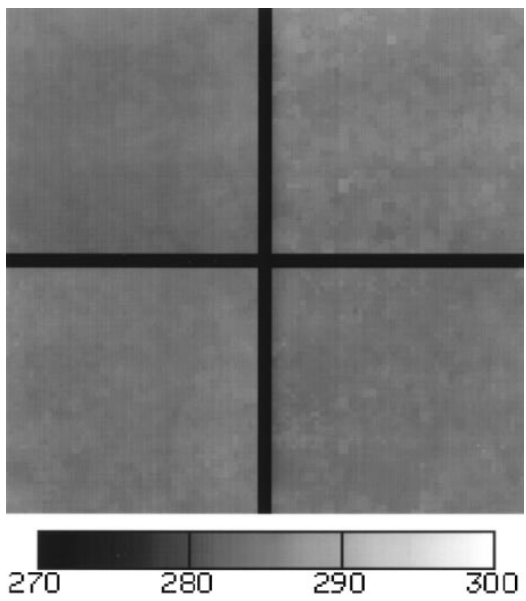

Fig. 11. SSM/I 85-V clear region cloud-removal composite images. From top left to bottom right: mean, second-highest value, MMA, and hybrid.

TABLE III

Pixel Value Averages and Standard Deviations of Each Algorithm Composite Image for the Cloudy and Clear Regions

\begin{tabular}{c|c|c||c|c}
\hline & \multicolumn{2}{|c||}{ Cloudy Region } & \multicolumn{2}{c}{ Clear Region } \\
\hline Algorithm & Ave. & St,d. & Ave. & Std. \\
\hline Mean & 279.8 & 3.259 & 284.4 & 0.480 \\
\hline Second Highest & 286.2 & 1.033 & 285.6 & 0.551 \\
\hline MMA & 284.7 & 0.973 & 285.1 & 0.509 \\
\hline Hybrid & 284.7 & 0.982 & 284.8 & 0.523 \\
\hline
\end{tabular}

possible to determine the bias in each image. However, we note that the mean image has the highest standard deviation. This is expected since the atmospherically distorted values were included in the mean. The second-highest value standard deviation is governed by the variance of the second-highest value only. As a result, this statistic is greatly improved over the mean image case. MMA yields the lowest standard deviation. The averaging contained in the MMA algorithm provides some additional noise reduction over the secondhighest value. The hybrid image has the same mean as MMA and a slightly higher standard deviation, indicating that the two algorithms performed similarly for the cloudy region. This shows that, as predicted, the hybrid algorithm effectively chooses MMA in cloudy regions.
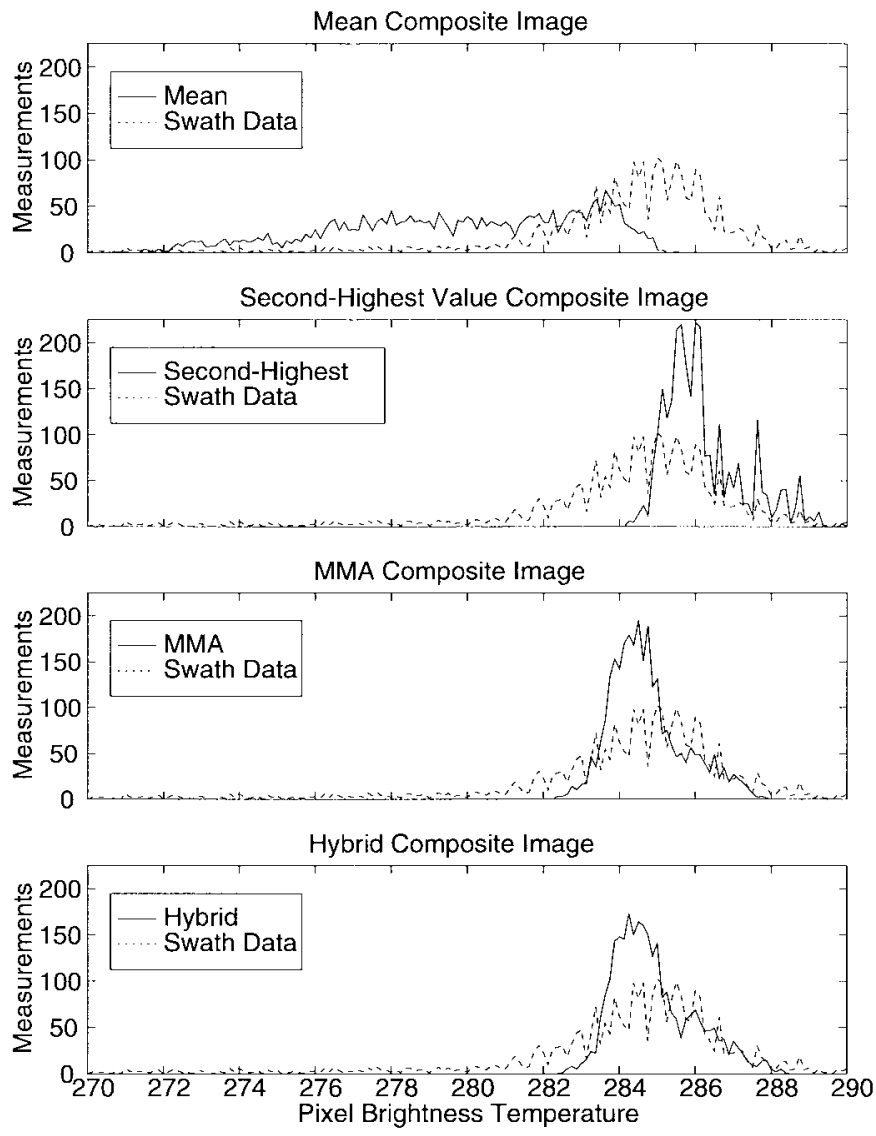

Fig. 12. SSM/I 85-GHz vertical channel cloudy region spatial brightness temperature distributions for each cloud-removal algorithm. The temporal and spatial measurement distribution has been plotted to provide a reference.

Fig. 11 shows the $85-\mathrm{V}$ channel composite images for a clear or distortion-free region. The average and standard deviation of all pixel values for each image are shown in Table III. Since we have chosen this region specifically because it apparently has no distortion problems, the mean image is unbiased - unlike the other algorithms' composite images. Hence, we can treat its average as the ground truth in this instance. With this in mind, the hybrid algorithm provides the best estimate to the true brightness temperature and is the least biased according to its average value. The secondhighest value is the most biased, with MMA falling in between. Thus, the hybrid provides the closest approximation to the "actual value," with MMA following closely behind. A closer examination of the standard deviation image (Fig. 4) reveals that some of the pixels in this area have values higher than the $1.25-\mathrm{K}$ threshold, indicating that some subtle temporal variations exist due to minor atmospheric distortions or small seasonal surface brightness temperature changes. Regardless, the hybrid algorithm provides the estimate with the least bias. As in the cloudy region, MMA has a lower standard deviation than the second-highest value. The hybrid noise level is only slightly higher than MMA.

The spatial pixel brightness temperature distributions for the composite images are plotted for both regions after each compositing algorithm is run. Figs. 12 and 13 present the results. Each of the distributions is plotted over the full temporal and spatial measurement distribution for that particular region. 

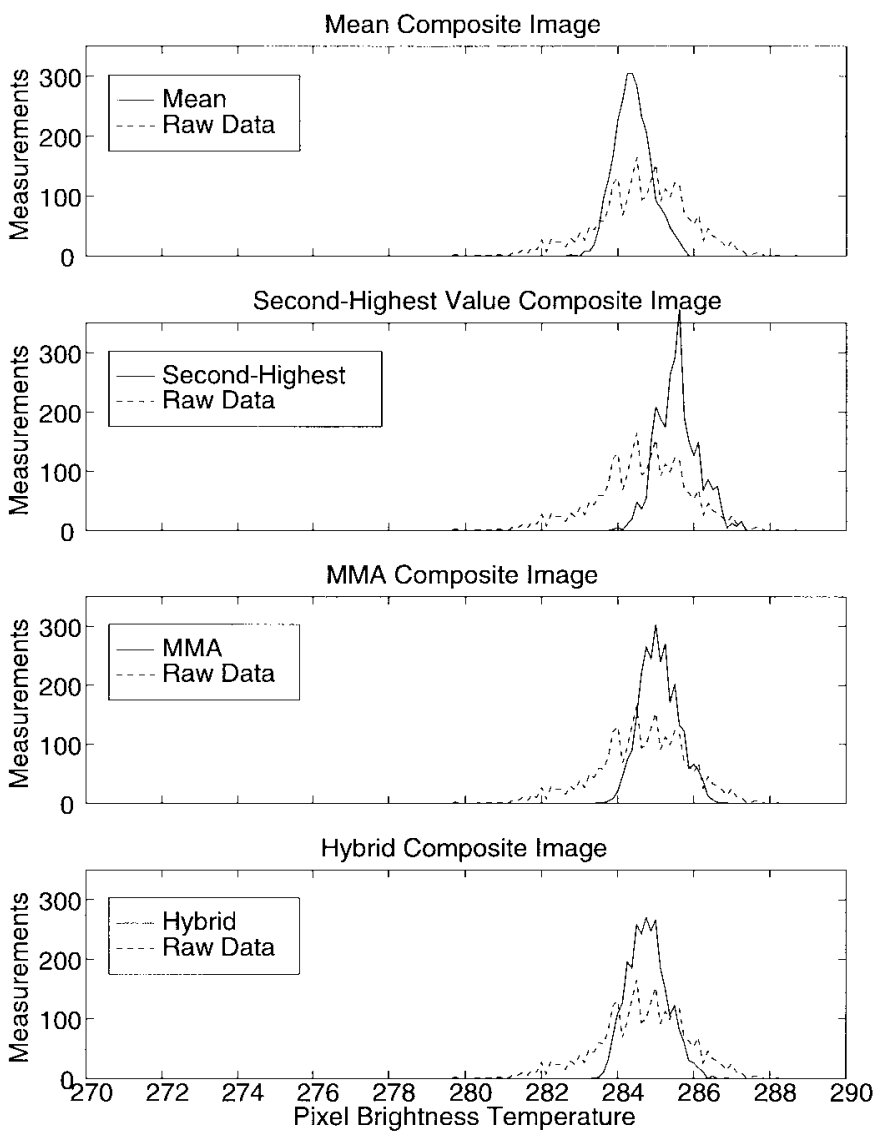

Fig. 13. SSM/I 85-GHz vertical channel clear region spatial brightness temperature distributions for each cloud-removal algorithm. The temporal and spatial measurement distribution has been plotted to provide a reference.

These help validate the assumptions on which the algorithms were based and provide a measure of algorithm performance.

Fig. 12 illustrates the algorithm composite image brightness temperature distributions of the cloudy region for the $85-\mathrm{GHz}$ channel. Clearly, the MMA and the hybrid most closely match the upper mode of the raw data distribution. Their similar performance indicates that hybrid primarily used the MMA algorithm in the presence of atmospheric distortion.

Distributions for the clear region are given in Fig. 13. The high bias inherent to the second-highest value method is once again evident. In the clear region, MMA also demonstrates some bias, although not as large. This is because MMA removes pixel values regardless of the actual presence of clouds or precipitation. On the other hand, the hybrid distribution has less bias and most closely matches the distribution of the raw data.

\section{CONCLUSION}

A comparison of several different methods (mean, secondhighest value, MMA, and hybrid) for creating cloud-free temporal composite surface brightness temperature images from SSM/I has been presented. Taking the mean is optimum in the absence of clouds and hydrometeors. The second-highest value algorithm removes distorted pixel brightness temperature measurements, but it has an inherent high bias in its brightness temperature distribution. The MMA algorithm more accurately estimates the desired value and has a lower variance; however, it may have an undesirable bias for instances in which no distortion is present. The hybrid algorithm combines the strengths of the mean and MMA algorithms. It contains a decision making routine that switches between the mean and MMA, depending on the apparent presence of clouds or precipitation. Simulations indicate that the hybrid algorithm more accurately approximates a pixel's actual brightness temperature for different distortion temperatures. Analysis of the composite image distributions also shows that the hybrid most closely approximates the upper mode of the real data distributions for the study region considered.

We note that in the presence of persistent atmospheric distortions, the distortion cannot be removed. However, the effects of the more heavily distorted pixels can still be reduced since MMA and the hybrid algorithms choose only the lessdistorted pixels in the averaging. It should also be noted that the algorithms can be optimized for specific study regions. Areas with different latitudes may be able to use shorter collection periods to gather the same number of measurements per pixel due to the satellite orbit geometry. The decision threshold for the hybrid can be tuned for specific regions according to the expected number of local atmospheric distortion events.

\section{ACKNOWLEDGMENT}

The SSM/I data used in this paper were made available courtesy of Dr. F. Wentz at Remote Sensing Systems [20].

\section{REFERENCES}

[1] B. Choudhury and C. Tucker, "Satellite observed seasonal and interannual variation of vegetation over the Kalahari, the great Victoria Desert, and the great Sandy Desert: 1979-1984," Remote Sens. Environ., vol. 23, pp. 233-241, 1987.

[2] M. R. Farrar and E. A. Smith, "Spatial resolution enhancement of terrestrial features using deconvolved SSM/I brightness temperatures," IEEE Trans. Geosci. Remote Sensing, vol. 30, pp. 349-355, Mar. 1992.

[3] J. D. Gibbons, Nonparametric Statistical Inference, 2nd ed. New York: Marcel Dekker, 1985.

[4] N. C. Grody, "Classification of snow cover and precipitation using the special sensor microwave/imager," J. Geophys. Res., vol. 94, pp. 7423-7435, Apr. 1991.

[5] J. P. Hollinger, J. L. Pierce, and G. A. Poe, "SSM/I instrument evaluation," IEEE Trans. Geosci. Remote Sensing, vol. 28, pp. 781-790, Sept. 1990.

[6] J. Hollinger et al., "Special sensor microwave/imager user's guide," Naval Res. Lab., Washington, DC, Dec. 14, 1987.

[7] J. Hollinger, "DMSP special sensor microwave/imager calibration/validation," Naval Res. Lab., Washington, DC, Final Rep., July 1989, vol. 1.

[8] T. J. Jackson and T. J. Schmugge, "Algorithm for the passive microwave remote sensing of soil moisture," in Microwave Radiometry and Remote Sensing Applications, P. Pampaloni, Ed. Utrecht, The Netherlands: (Zeist)VSP, 1989, pp. 3-17.

[9] A. K. Jain, Fundamentals of Digital Image Processing. Englewood Cliffs, NJ: Prentice-Hall, 1989.

[10] A. S. Jones and T. H. V. Haar, "Passive microwave sensing of cloud liquid water over land regions," J. Geophys. Res., vol. 95, pp. 16673-16 683, Sept. 1990.

[11] D. G. Long and D. L. Daum, "Spatial resolution enhancement of SSM/I data," IEEE Trans. Geosci. Remote Sensing, vol. 36, pp. 407-417, Mar. 1998.

[12] M. J. McFarland, R. L. Miller, and C. M. U. Neale, "Land surface temperature derived from the SSM/I passive microwave brightness temperatures," IEEE Trans. Geosci. Remote Sensing, vol. 28, pp. 839-845, Sept. 1990.

[13] P. M. Narenda, "A separable median filter for image noise smoothing," IEEE Trans. Pattern Anal. Machine Intell., vol. PAMI-3, pp. 20-29, Jan. 1981. 
[14] C. M. U. Neale, M. J. McFarland, and K. Chang, "Land-surface-type classification using microwave brightness temperatures from the special sensor microwave/imager," IEEE Trans. Geosci. Remote Sensing, vol. 28, pp. 829-838, Sept. 1990.

[15] P. Pampaloni and S. Paloscia, "Microwave emission and plant water content: A comparison between field measurement and theory," IEEE Trans. Geosci. Remote Sensing, vol. GE-24, pp. 900-904, Nov. 1986.

[16] S. D. Prince and B. J. Choudhury, "Interpretation of nimbus-7 $37 \mathrm{GHz}$ microwave brightness temperature in semi-arid Southern Africa," Int. J. Remote Sensing, vol. 10, no. 10, pp. 1643-1661, 1989.

[17] R. W. Spencer, H. M. Goodman, and R. E. Hood, "Precipitation retrieval over land and ocean with the SSM/I: Identification and characteristics of the scattering signal," J. Atmos. Ocean. Technol., vol. 6, pp. 254-273, Apr. 1989.

[18] R. H. Thomas, R. A. Bindschadler, R. L. Cameron, F. D. Carsey, B. Holt, T. J. Hughes, C. W. M. Swithinbank, I. M. Whillans, and H. J. Zwally, "Satellite remote sensing for ice sheet research," NASA Tech. Memo. 86233, 27, 1985.

[19] F. T. Ulaby, R. K. Moore, and A. K. Fung, Microwave Remote Sensing-Active and Passive, vol. 1. Norwood, MA: Artech House, 1981.

[20] F. J. Wentz, "User's manual SSM/I antenna temperature tapes," Remote Sensing Syst., Santa Rosa, CA, 1991.

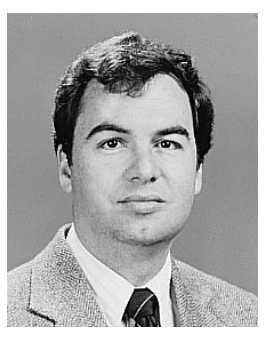

David G. Long (S'80-M'82-SM'98) received the $\mathrm{Ph} . \mathrm{D}$. degree in electrical engineering from the University of Southern California, Los Angeles, in 1989.

He was with NASA's Jet Propulsion Laboratory (JPL), Pasadena, CA, from 1983 to 1990, where he developed advanced radar remote-sensing systems. While at JPL, he was the Senior Project Engineer on the NASA Scatterometer (NSCAT) Project. NSCAT was launched aboard the Japanese Advanced Earth Observing System (ADEOS) in August 1996. He is currently an Associate Professor in the Electrical and Computer Engineering Department, Brigham Young University, Provo, UT, where he teaches upper division and graduate courses in communications, microwave remote sensing, radar, and signal processing. He is the Principle Investigator on several NASAsponsored interdisciplinary research projects in remote sensing, including innovative radar systems, spaceborne scatterometry of the ocean and land, and modeling of atmospheric dynamics. He is a member of the NSCAT Science Working Team. He has numerous publications in signal processing and radar scatterometry. His research interests include microwave remote sensing, radar theory, space-based sensing, estimation theory, computer graphics, signal processing, and mesoscale atmospheric dynamics.

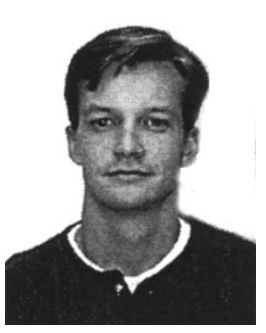

Quinn P. Remund received the B.S. degree in electrical and computer engineering from Brigham Young University (BYU), Provo, UT, in December 1996. He is currently pursuing the Ph.D. degree at BYU, where he is a recipient of the Rocky Mountain NASA Space Grant Consortium Research Fellowship.

$\mathrm{He}$ has been a member of the Microwave Earth Remote Sensing Group, BYU, since 1995. His current research interests focus on theory and applications of satellite microwave remote sensing of the polar regions using multifrequency and multipolarizaton measurements.

Douglas L. Daum, photograph and biography not available at the time of publication. 